\title{
Genome shuffling: a method to improve biotechnological processes
}

\author{
Katarzyna LeJA*, Kamila Myszka, Katarzyna CzaczyK \\ Department of Biotechnology and Food Microbiology, Poznan University of Life Sciences, Poznań, Poland \\ *Corresponding author: katleja@up.poznan.pl
}

\begin{abstract}
Genome shuffling is widely used for increasing the production of metabolites by bacterial strains, improving substrate uptake as well as enhancing strain tolerance. This technique combines the advantage of multiparental crossing allowed by DNA shuffling together with the recombination of entire genomes normally associated with conventional breeding, or through protoplast fusion that increases the recombination process. The method of genome shuffling was first presented by Stemmer and co-workers in 2002 when it was used to improve the production of tylosin by Streptomyces fradiae. Nowadays, it is used in many experiments for increasing the production of metabolites by bacterial strains, improving substrate uptake, and enhancing strain tolerance. Genome shuffling is a major milestone in the strain-improvement technology and metabolic engineering.
\end{abstract}

Key words: bacteria strains, genome shuffling, mutagenesis, protoplast fusion, strain-improvement technology

\section{Introduction}

An important objective of a biotechnological research is the engineering of microbial cells for the production of industrially valuable metabolites. The main objective of research into effective technologies is to improve bacteria strains that are able to produce metabolites, and which will find application in industries such as chemical, food, oenology, pharmaceutical, and biofuel (Gong et al., 2009). To achieve that, a classical strain improvement method is usually employed. This technique is an asexual process ased on sequential random mutagenesis and screening. A promising microorganism is mutagenized to produce a diverse library of random mutants. Screening enables selecting individual strains with an improved relevant phenotype. However, this method seems to be a laborious and time-consuming process, which is its main disadvantage (del Cardayré, 2005; Gong et al., 2009), and genome shuffling seems to be a better way of improving industrially important strains. This technique combines the advantage of multiparental crossing, possible through DNA shuffling, along with the recombination of entire genomes which is normally associated with conventional breeding or through a protoplast fusion that, increases recombination (Zhang et al., 2002; del Cardayré, 2005; Cheng et al., 2009). Moreover, genome shuffling can accelerate directed evolution by facilitating recombination between the members of a diversely selected population (Zhang et al., 2002; Cheng et al., 2009). Genome shuffling is similar to the classical strain improvement in that it is a cycle of genomic diversification and screening for improved strains. The main difference between these two techniques is that genome shuffling process is sexual and whole populations of improved strains are evolved, as opposed to the classical strains improvement (del Cardayré and Powell, 2003). What is more, the technique of genome shuffling is not limited to the microbe which has a clear genetic background (Gong et al., 2009). Currently, genome shuffling is one of the most efficient methods for the evolution of strains toward desirable phenotypes. Different genes which are associated with the production of metabolites can be recombined during several rounds of genome shuffling and consequently desirable phenotypes can be obtained (Jin et al., 2009).

The method of genome shuffling was first presented by the Stemmer and co-workers in 2002, when the whole-genome shuffling was described. It was demonstrated that genomic recombinations within a population of bac- 
teria can efficiently generate combinatorial libraries of new strains. Many of those new strains show marked improvements in the selected phenotype. Genome shuffling has been used to improve the production of polyketide antibiotic (tylosin) by Streptomyces fradiae (Zhang et al., 2002). After only two rounds of genome shuffling, strains were obtained with production levels comparable to those of strains which had been developed over two decades of via 20 rounds of classical strains improvement methods (Lutz and Bornscheuer, 2009). Genome shuffling was regarded as a major milestone in the strain-improvement technology and metabolic engineering (Stephanopoulos, 2002). Nowadays, the technique of genome shuffling is used to significantly improve the quality of industrially important microbiological phenotypes (Gong et al., 2009). It was successfully used, inter alia, for improving the acid tolerance in Lactobacillus (Patnaik et al., 2002; Wang et al. 2007), enhancing the resistance of Spingobium chlorophenolicum to the toxicity of pentachlorophenol (Dai and Copley, 2004), increasing resistance to (2S, 3R)-hydroxycitric acid in Streptomyces sp. (Hida et al., 2007), and increasing the efficiency of the 1.3-propanediol production (Otte et al., 2009).

Genome shuffling is a more convenient method when compared to other molecular breeding techniques and can be easily popularized. The application of this method does not require expensive facilities. The cost of genome shuffling is not high, either. Importantly, this technique is easy to handle and can be used in most laboratories (Gong et al., 2009).

\section{Basic of genome shuffling}

Protoplast fusion is a method which leads to genome shuffling. It has been used to modify the phenotypic traits of a variety of prokaryotic and eukaryotic cells since the late 1970s (Hopwood et al., 1977; Scheinbach, 1983; Iwata et al., 1986; Petri and Schmidt-Dannert, 2004; Gong et al., 2009). Protoplast fusion was successfully achieved first in animals, and later in plants (Rai and Rai, 2006). Protoplast from different species, and even from different kingdoms, can be successfully fused. This indicates a very broad applicability of this technique in cell engineering (Verma et al., 1992; Rassoulzadegan et al., 1982; Petri and Schmidt-Dannert, 2004). In contrast to other established genetic manipulation techniques, a high frequency of recombination can be achieved by fusing complete protoplast genomes (Hopwood and Wright, 1979; Petri and Schmidt-Dannert, 2004; Gong et al,. 2009). Thus, although many advanced technologies based on genetic manipulation have been developed over the years, protoplast fusion continues to be widely applied for phenotypic improvement (Gong et al., 2009).

Despite the fact that genome shuffling originated from protoplast fusion, it is a different method when compared to the same. Traditional protoplast fusion is the fusion between two cells with different genetic traits. It leads to a stable recombinant with the combination of the genetic traits of both parents. In this process, the recombination results from only two parents per generation. Genome shuffling, on the contrary, is the recombination between multiple parents of each generation, and several rounds of genome fusion are carried out. As a result, the final improved strains involve the genetic trait from multiple initial strains. Genome shuffling is quite cost effective; its application does not require any expensive facility and can be easily employed in most laboratories (Gong et al., 2009). Most importantly, shuffled strains are not considered as genetically modified organisms and can therefore be used in the food industry (Zhang et al., 2002; Ahmed, 2003; Petri and SchmidtDannert, 2004).

\section{Strategies for genome shuffling}

The procedure of genome shuffling consists of two main steps: construction of the parental library protoplast fusion, and the selection of the desired phenotype. The general scheme of genome shuffling is presented in Figure 1. First of all, the parent strain library must be constructed. To achieve that, the initial strain is engineered to generate more genotypes. The desirable strains are then collected to form the parental library for protoplast fusion. Three different mutagens are mainly used for mutagenesis. They are: ethyl methanesulfonate, $\mathrm{N}$-methyl-N" '-nitro-N-nitrosoguanidine, and ultraviolet radiation (Wang et al., 2007; Cheng et al., 2009; Gong et al., 2009; Jin et al., 2009; Otte et al., 2009). An important feature of the process is that if the selection of the parental strain is not suitable, the desired phenotype will not be obtained (Hida et al., 2007; Gong et al., 2009). The next step of genome shuffling is protoplast fusion. The cells are re-suspended in a buffer containing 


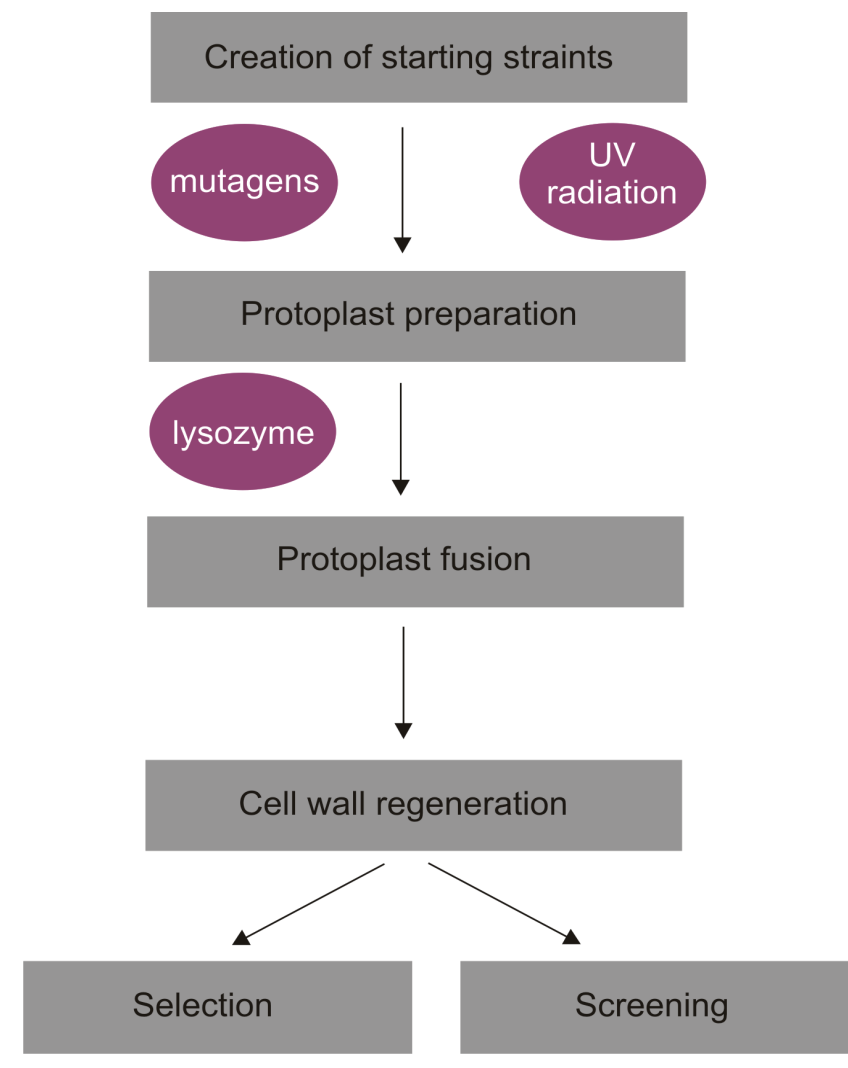

Fig. 1. A general scheme of the genome shuffling process

lysozyme (John et al., 2008; Otte et al., 2009) or other enzymes such as snailase (Cheng et al., 2009), and following that protoplasts are obtained and aggregated by centrifugation (Otte et al., 2009). Then, an equal numbers of protoplasts from the mutants are mixed, divided equally into two parts, and inactivated. One part is incubated at a high temperature $\left(50^{\circ} \mathrm{C}\right.$ or $60^{\circ} \mathrm{C}$ ) (Hopwood and Wright, 1979; Wang et al., 2007; Cheng et al., 2009; Otte et al., 2009), and the other part is irradiated by UV. The killed protoplasts are grouped together and fused in a system containing $35 \%$ PEG6000 and $0.1 \% \mathrm{CaCl}_{2}$ at $35^{\circ} \mathrm{C}$ for $40 \mathrm{~min}$. Next, the fused protoplasts are centrifuged, washed twice, re-suspended in $10 \mathrm{ml}$ buffer, serially diluted and regenerated on the regeneration medium supplemented with, among others, glucose, bovine serum albumin, casein hydrolysate $\mathrm{MgCl}_{2}, \mathrm{CaCl}_{2}$, and gelatin for six days at $30^{\circ} \mathrm{C}$. (John et al., 2008; Otte et al., 2009). Strains from the regenerated protoplasts are pooled, resulting in the strain library for the second round fusion. These strains grow as a population and are used for preparing protoplasts which are similarly fused and regenerated. This process may be repeated several times. Protoplast fusion with multiparental strains ena- bles efficient shuffling of a phenotypically selected population for the purpose of strain evolution (Petri and Schmidt-Dannert, 2004; Gong et al., 2009). The final step of genome shuffling is the selection of desired phenotypes. The desired phenotypes are obtained from the populations resulting from protoplast fusion through the screening process. The classical method for screening yield improved strain is determined by physiological or biochemical characters such as hydrolysis zone, clear zones (John et al., 2008), inhibition zone in the agar plates. However, some novel methods for the isolation of the desired strains have been described. For example, in the work of Hida et al. (2007), an addition of analogs of products was used as an efficient method for isolating high product strains. An analog of hydroxycitric acid (trans-epoxy aconitic acid) was utilized to screen the desirable bacteria metabolite - hydroxycitric acid. Trans-epoxy aconitic acid inhibited the regeneration of non-fused protoplasts, resulting in selective screening of shuffled strains (Gong et al., 2009).

\section{Application of genome shuffling}

Genome shuffling is applied in order to increase the production of metabolites by bacterial strains (Zhang et al., 2002; Chen et al., 2004; Bode and Muller, 2006; Zirkle et al., 2007; Liang and Guo, 2007; Lin et al., 2007; Otte et al., 2009; Zhang et al., 2010), with the aim to improve substrate uptake (Dai and Copley, 2004; Kumar 2007; John et al., 2008), and enhance strain tolerance (Patnik et al., 2002; Kawahata et al., 2006; Graves et al., 2007; Yu et al., 2007; Wei et al., 2008; Xu et al., 2008; John et al., 2008; Gong et al., 2009; Shi et al., 2009).

\section{An improvement of substrate uptake}

In this category of genome shuffling the application of a number of experiments is described, such as, the first application of this technique - rapid improvement of Streptomyces fradiae which produces tylosin (Zhang et al., 2002; Xu et al., 2011). A desirable effect was obtained after two rounds of genome shuffling. This effect was comparable with the results obtained after twenty years of research on mutation and screening (Gong et al., 2009). Two years later, in 2011, a riboflavin-producing strain of Bacillus subtilis was successfully improved by genome shuffling. Also in that case merely two rounds of genome shuffling were optimal. As a result, bacteria strains were obtained which produce 
about $100 \%$ more riboflavin when compared with the original strain (Chen et al., 2004). Xu et al. (2006) used genome shuffling to improve Actinoplanes teichomyceticus strain that produced teicoplanin. In one year, the potency of the improved strain increased to up to $65.3 \%$. In 2007 , the genome shuffling was used successfully in eukaryotic microorganisms. It was used for improving the lipase production of Penicillium expansum. Two strains, a lipase-producing mutant strain - Penicillium expansum FS8486 and a wild type Aspergillus tamarii FS-132 isolated from the soil of a volcano in Xinjiang in China, were used as parental strains. After two rounds of genome shuffling, daughter strains with desirable features were screened. Lipase activity in one of the daughter strains was increased by $317 \%$ over the starting strain FS8486 (Lin et al., 2007). In the same year, Gong et al. (2007) mutated the epothilone that produced myxobacterium Saccharomyces cellulosum strain So0157-2 to improve the production of epothilones, highly promising prospective anticancer agents. The epothilone production of fusants increased about 130 times after two rounds of genome shuffling as compared to the starting strain. Further research during the same year focused on genome shuffling of Streptomyces sp. strains which are capable of hydroxycitric acid production. Hydroxycitric acid inhibits pancreatic $\alpha$-amylase and intestine $\alpha$-glucosidase, leading to the reduction of carbohydrate metabolism. The initial Streptomyces population was generated by N-methyl- ${ }^{\prime \prime}$ nitro-N-nitrosoguanidine treatment of the spores, and an improved population producing fivefold more acid was obtained after three rounds of genome shuffling (Hida et al., 2007). Jin et al. (2009) used the genome shuffling technique to enhance the production of spinosad (a mixture of secondary metabolites) in Saccharopolyspora spinosa. Spinosad is used in agriculture as a potent insect control agent with exceptional safety toward non-target organisms. S. spinosa starting strain was mutagenized by nitrosoguanidine and UV radiation. After four rounds of genome shuffling, a high yielding strain was isolated. The production of spinosad increased by $200 \%$ and $436 \%$ in comparison with that of the highest parent strain and the original strain, respectively. Recently, in 2009 and 2010, two experiments were carried out in which the genome shuffling technique was used to improve 1,3-propanediol and vitamin $B_{12}$ production (Otte et al., 2009; Zhang et al., 2010). In the first experiment, in 2009, genome shuffling was applied to Clostridium diolis DSM
15410 to improve the production of 1,3-propanediol (Otte et al., 2009) which is a valuable chemical intermediate and may be poten-tially used in the facture of polymers (among others, polyesters, polyethers, polyurethanes), cosmetics, foods, lubricants, medicines, and as an intermediate for the synthesis of heterocyclic compounds (Leja et al., 2011; Drożdżyńska et al., 2011). Otte et al. (2009) used N-Methyl-N"-nitro-N-nitrosoguanidine for mutagenesis of Clostridium diolis. The four rounds of genome shuffling of obtained mutants with desirable phenotypes were performed. A significant improvements were observed, with one strain attaining a 1,3-propanediol volumetric yield of $85 \mathrm{~g} / \mathrm{l}$. That result represented an $80 \%$ improvement compared to the yield from the parental wild-type strain. In 2010 genome shuffling was used to improve Propionibacterium shermanii strain which is capable of vitamin $\mathrm{B}_{12}$ production. Two mutagenesis N-Methyl- $\mathrm{N}^{\prime \prime}$-nitro-N-nitrosoguanidine and methylsulfonate were used. After 96 hours, the genomed shuffled strain from the first generation produced about $61 \%$ improvement of vitamin $B_{12}$ over the parent strain. Moreover, the genomed shuffled strain showed better growth than the parent strain (Zhang et al., 2010). A new application of genome shuffling was presented in 2011. Xu et al. (2011) used genome shuffling to enhance cellulase production by Trichoderma viride. The initial strain mutant populations were generated by UV radiation, low-energy ion beam implantation and atmospheric pressure non-equilibrium discharge plasma. The shuffled strain, obtained after two rounds of genome shuffling, exhibited a total cellulase activity of $4.17 \mathrm{U} / \mathrm{g}$ which was 1.97-fold higher that of wild-type strain Trichoderma viride.

\section{An improvement of substrate conversion}

Dai and Copley (2004) improved the degradation of pentachlorophenol by Sphingobium chlorophenolicum by the genome shuffling technique. Sphingobium chlorophenolicum can degrade pentachlorophenol. These bacteria cannot tolerate high levels of this highly toxic anthropogenic pesticide. Moreover, the degradation of pentachlorophenol is very slow. Scientists have obtained several strains that degrade pentachlorophenol faster and tolerate its higher levels than the wild-type strain. After the third round of shuffling, several strains capable of growing on the high concentration of pentachlorophenol were obtained. Additionally, some strains were 
capable of completely degrading $3 \mathrm{mM}$ pentachlorophenol, whereas no degradation could be achieved by the wild type strain. Analysis of several improved strains suggest that the improved phenotypes lead, due to various combinations of mutations, to an enhanced growth rate, constitutive expression of the pentachlorophenol degradation genes, and enhanced resistance to the toxicity of pentachlorophenol and its metabolites. John et al. (2008) used genome shuffling for producing L-lactic acid from starchy wastes. As a parental strain Lactobacillus delbrueckii and Bacillus amyloliquefaciens were employed. After three rounds of genome shuffling a mutant strain capable of utilizing the liquefied cassava bagasse starch directly with minimum nutrient supplementation for lactic acid production was obtained. The result of this experiment, lactic acid yield of $40 \mathrm{~g} / \mathrm{l}$ obtained with a productivity of $0.42 \mathrm{~g} / \mathrm{l} / \mathrm{h}$ and $96 \%$ conversion of starch to lactic acid, is comparable and even better than the reported single step conversion of starch to lactic acid.

\section{The enhancement of strain tolerance}

Genome shuffling was used for increasing acid and glucose tolerance in Lactobacillus (Gong et al., 2009). The results of these experiments have been described in a number of papers published by, inter alia, Patnik et al. (2002), Wang et al. (2007), John et al. (2008), and Yu et al. (2007, 2008). Lactic acid is an environment-friendly chemical with a wide range of industrial applications that are the focus of attention and concern of researchers and biotechnologists worldwide. The economic production of lactic acid can be achieved by exploiting various biotechnological techniques. Among those, there is the improvement of microorganism strains for industrially desirable characteristics such as high yield and productivity of lactic acid and the ability to grow at low $\mathrm{pH}$ and to utilize complex agro-industrial wastes (Rojan et al., 2010). Patnik et al. (2002) used genome shuffling to improve the acid tolerance of a poorly characterized industrial strain of Lactobacillus. Scientists in that experiment used classical strain-improvement methods to generate populations with subtle improvements in $\mathrm{pH}$ tolerance, and then shuffled these populations through protoplast fusion. They identified new shuffled lactobacilli that grow at substantially lower $\mathrm{pH}$ than the wildtype strain does. Moreover, shuffled strains that produced three-fold more lactic acid than the wild type at $\mathrm{pH}$ 4.0 have also been identified. Wang et al. (2007) im- proved the acid tolerance and volumetric productivity of an industrial strain Lactobacillus rhamnosus ATCC 11443. They used ultraviolet irradiation and N-Methyl$\mathrm{N}^{\prime \prime}$-nitro-N-nitrosoguanidine for mutagenesis. After that, five strains with subtle improvements in $\mathrm{pH}$ tolerance and volumetric productivity were obtained from the populations generated by mutagenesis. Next, they were subjected to protoplast fusion. After three rounds of genome shuffling, four strains that could grow at $\mathrm{pH} 3.6$ were obtained. Scientists observed 3.1- and 2.6-fold increases in the lactic acid production and the cell growth of the best performing at $\mathrm{pH} 3.8$, respectively. Yu et al. (2008) used genome shuffling to improve the glucose tolerance of Lactobacillus rhamnosus ATCC 11443, the same strain used by Wang et al. (2007), and to with simultaneously enhance the L-lactic acid production. The starting population was also generated by ultraviolet irradiation and nitrosoguanidine. As a result of their experiment they presented the lactic acid production, cell growth, and glucose consumption of the best performing strain from the second round of genome shuffled populations $71.4 \%, 44.9 \%$, and $62.2 \%$ higher than those of the wild type. The genome shuffling technique was also used for improving acetic acid tolerance in Candida krusei (Wei et al., 2008). The better mutant was isolated and selected after four rounds of genome shuffling.

Selected strain had a higher viability in medium with acetic acid and grew better with acetic acid than the parent strain and improved multiple stress tolerance to ethanol, $\mathrm{H}_{2} \mathrm{O}_{2}$, heat, and freeze-thaw. Furthermore, the obtained mutant demonstrated higher ethanol production than the parent strain in a medium with or without acetic acid. The DNA content of mutant was similar to its parent strains in the genome shuffling. Shi et al. (2009) described the application of genome shuffling to improve thermotolerance in Saccharomyces cerevisiae. After three rounds of genome shuffling the best performing strain was obtained. The improved $S$. cerevisiae strain could tolerate $25 \%(\mathrm{v} / \mathrm{v})$ ethanol stress and produce $9.95 \%(\mathrm{w} / \mathrm{v})$ ethanol. Moreover, the best strain could grow on plate culture at $55^{\circ} \mathrm{C}$.

Genome shuffling has many applications in increasing the phenotypes in industrially important bacteria strains. However, this technique can also increase our knowledge of metabolic network and their regulation. Genome shuffling offers advantages of simultaneous changes at different positions throughout the entire genome. 


\section{Conclusions}

Genome shuffling is a recent breakthrough in shuffling technology that combines the advantage of multiparental crossing allowed by DNA shuffling with the recombination of entire genomes normally associated with conventional breeding. It is a better technology for engineering phenotypes at the whole genome level. In many experiments, genome shuffling has been demonstrated as an effective method for producing improved bacterial strains. This modern application of protoplast fusion has resulted in about 40 research articles in this field till now. One of the reasons that this technique is often used owes to its simplicity. What is more, it does not require expensive genetic tools.

\section{Acknowledgments}

This paper has been prepared within the framework of project no.01.01.02-00-074/09, co-funded by The European Union from The European Regional Development Fund within the framework of the Innovative Economy Operational Programme 2007-2013.

\section{References}

Ahmed F.E. (2003) Genetically modified probiotics in foods. Trends Biotechnol. 21: 491-497.

Bode H.B., Muller R. (2006) Analysis of myxobacterial secondary metabolism goes molecular. J. Ind. Microbiol. Biotechnol. 33: 577-588.

Chen T., Wang J.Y., Zhou S.Q., Chen X., Ban R., Zhao X.M. (2004) Trait improvement of riboflavin producing Bacillus subtilis by genome shuffling and metabolic flux analysis. J. Chem. Ind. Eng. 55: 1842-1848.

Cheng Y., Song X., Qin Y., Qu Y. (2009) Genome shuffling improves production of cellulose by Penicillium decumbens JU-A10. J. Appl. Microbiol. 107: 1837-1846.

Dai M.H., Copley S.D. (2004) Improved production of ethanol by novel genome shuffling in Saccharomyces cerevisiae. Appl. Environm. Microbiol. 70: 2391-2397.

del Cardayré S.B., Powell K. (2003) DNA shuffling for whole cell engineering. In: Handbook of Industrial Cell Culture Mammalian, Microbial, and Plant Cells, ed. Vinci V.A., Parekh S.R., Humana Press, New Jersey, p. 476-480.

del Cardayré S.B. (2005) Developments in strain improvement technology evolutionary engineering of industrial microorganisms through gene, pathway, and genome shuffling. In: Natural Products Drug Discovery and Therapeutic Medicine, ed. Zhang L., Demain A.L., Humana Press, New York, p. 117-119.

Drożdżyńska A., Leja K., Czaczyk K. (2011) Biotechnological production of 1,3-propanediol from crude glycerol. BioTechnologia 92(1): 92-100.
Gong G.L., Sun X., Liu X.L., Hu W., Cao W.R., Liu H., Liu W.F., Li Y.Z. (2007) Mutation and a high-throughput screening method for improving the production of Epothilones of Sorangium. J. Ind. Microbiol. Biotechnol. 34: 615-623.

Gong J., Zheng H., Wu Z., Chen T., Zhao X. (2009) Genome shuffling: Progress and applications for phenotype improvement. Biot. Adv. 27: 996-1005.

Graves T., Narendranath N.V., Dawson K., Power R. (2007) Interaction effects of lactic acid and acetic acid at different temperatures on ethanol production by Saccharomyces cerevisiae in corn mash. Appl. Microbiol. Biotechnol. 73: $1190-1196$.

Hida H., Yamada T., Yamada Y. (2007) Genome shuffling of Streptomyces sp. U121 for improved production of hydroxycitric acid. Appl. Microbiol. Biotechnol. 73: 1387-1393.

Hopwood D.A., Wright H.M., Bibb M.J., Cohen S.N. (1977) Genetic recombination through protoplast fusion in Streptomyces. Nature 268: 171-174.

Hopwood D.A., Wright H.M. (1979) Factors affecting recombinant frequency in protoplast fusions of Streptomyces coelicolor. J. Gen. Microbiol. 111: 137-143

Iwata M., Mada M., Ishiwa H. (1986) Protoplast fusion of Lactobacillus fermentum. Appl. Environ. Microbiol. 52: 392-393.

Jin Z.H., Xu B., Lin S.Z., Jin Q.C., Cen P.L. (2009) Enhanced production of spinosad in Saccharomyces spinosa by genome shuffling. Appl. Biochem. Biotechnol. 159: 655-663.

John R.P., Gangadharan D., Nampoothiri K.M. (2008) Genome shuffling of Lactobacillus delbueckii mutant and Baccillus amyloliquefaciens through protoplasmic fusion for L-lactic production from starchy wastes. Biores. Technol. 99: 8008-8015.

Kawahata M., Masaki K., Fujii T., Iefuji H. (2006) Yeast genes involved in response to lactic acid and acetic acid: acidic conditions caused by the organic acids in Saccharomyces cerevisiae cultures induce expression of intracellular metal metabolism genes regulated by Aft1p. FEMS Yeast Res. 6: 924-936.

Kumar M. (2007) Improving polycyclic aromatic hydrocarbons degradation by genome shuffling. Asian J. Microbiol. Biotechnol. Environ. Sci. 9: 145-149.

Leja K., Czaczyk K., Myszka K. (2011) 1,3-Propanediol synthesis by biotechnological way using Clostridium ssp. African J. Biotech. (in press).

Liang H.Y., Guo Y. (2007) Whole genome shuffling to enhance activity of fibrinolytic enzyme producing strains. China Biotechnol. 27: 39-43.

Lin J., Shi B.H., Shi Q.Q., He Y.X., Wang M.Z. (2007) Rapid improvement in lipase production of Penicillium expansum by genome shuffling. Chin. J. Biotechnol. 23(4): 672-676.

Lutz S., Bornscheuer U.T. (2009) Biosynthetic Pathway Engineering Strategies. In: Protein Engineering Handbook, Willey-VCH Verlag GmbH\&Co, Weinheim, p. 862-864.

Otte B., Grunwaldt E., Mahmoud O., Jennewein S. (2009) Genome shuffling in Clostridium diolis DSM 15410 for improved 1,3-propanediol production. Appl. Environm. Microbiol. 75: 7610-7616. 
Patnaik R., Louie S., Gavrilovic V., Perry K., Stemmer W.P., Ryan C.M. et al. (2002) Genome shuffling of lactobacillus for improved acid tolerance. Nat. Biotechnol. 20: 707-712.

Petri R., Schmidt-Dannert C. (2004) Dealing with complexity: evolutionary engineering and genome shuffling. Curr. Opin. Biotechnol. 15: 298-304.

Rai N., Rai M. (2006) Reproduction, Pollen Biology, and Pollen Biotechnology. In: Heterosis breeding in vegetable crops, ed. Kalloo G., New India Publishing Agency, p. 184-185.

Rassoulzadegan M., Binetruy B., Cuzin F. (1982) High frequency of gene transfer after fusion between bacteria and eukaryotic cells. Nature 295: 257-259.

Rojan P., John G.S., Pandey A.A., Nampoothiri K.M. (2010) Genome shuffling: A new trend in improved bacterial production of lactic acid. Ind. Biotechnol. 6: 164-169.

Scheinbach S. (1983) Protoplast fusion as a means of producing new industrial yeast strains. Biotechnol. Adv. 1: 289-300.

Shi D.J., Wang C.L., Wang K.M. (2009) Genome shuffling to improve thermotolerance, ethanol tolerance and ethanol productivity of Saccharomyces cerevisiae. J. Ind. Microbiol. Biotechnol. 36: 139-147.

Stephanopoulos G. (2002) Metabolic engineering by genome shuffling: Nat Biotechnol. 20: 666-668.

Verma V., Qazi G.N., Parshad R. (1992) Intergeneric protoplast fusion between Gluconobacter oxydans and Corynebacterium species. J. Biotechnol. 26: 327-330.

Wang Y., Li Y., Pei X., Yu L., Feng Y. (2007) Genome-shuffling improved acid tolerance and L-lactic acid volumetric productivity in Lactobacillus rhamnosus. J. Biotechnol. 129: 510-515.

Wei P.Y., Li Z.L., He P., Lin Y., Jiang N. (2008) Genome shuffling in the ethanologenic yeast Candida krusei to improve acetic acid tolerance. Biotechnol. Appl. Biochem. 49: $113-120$.

Xu B., Jin Z.H.,Wang H.Z., Jin Q.C., Jin X., Cen P.L. (2008) Evolution of Streptomyces pristinaespiralis for resistance and production of pristinamycin by genome shuffling. Appl. Microbiol. Biotechnol. 80: 261-267.

Xu B., Wang M.R., Xia Y., Yang K., Zhang C.Y. (2006) Improvement of the output of teicoplanin by genome shuffling. Chin. J. Antibiot. 31: 237-242.

Xu F., Jin H., Li H., Tao L., Wang J., Lv J., Chen S. (2011) Genome shuffling of Trichoderma viride for enhanced cellulase production. Ann. Microbiol. 5: 176-191.

Yu L., Lei T., Pei X.L., Liu J.S. (2007) Application of genome shuffling in enhancing L-lactic acid production by improving glucose tolerance of Lactobacillus rhamnosus. Food Sci. 28: 369-373.

Yu L., Pei X.L., Lei T., Wang Y., Feng Y. (2008) Genome shuffling enhanced L-lactic acid production by improving glucose tolerance of Lactobacillus rhamnosus. J. Biotechnol. 134: 154-159.

Zhang Y., Liu J.Z., Huang J.S., Mao Z.W. (2010) Genome shuffling of Propionibacterium shermanii for improving vitamin $B_{12}$ production and comparative proteome analysis. J. Biotechnol. 148: 139-143.

Zhang Y.X., Perry K., Vinci V.A., Powell K., Stemmer W.P., del Cardayré S.B. (2002) Genome shuffling leads to rapid phenotypic improvement in bacteria. Nature 415: 644646.

Zirkle R., Ligon J.M., Molnar I. (2004) Heterologous production of the antifungal polyketide antibiotic soraphen $A$ of Sorangium cellulosum So ce26 in Streptomyces lividans. Microbiology-Sgm. 150: 2761-2774. 